\title{
Block Jacobi for discontinuous Galerkin discretizations: no ordinary Schwarz methods
}

Martin J. Gander ${ }^{1}$ and Soheil Hajian ${ }^{1}$

\section{Introduction}

We study in this paper block Jacobi iterations for matrix problems obtained by discontinuous Galerkin (DG) discretizations. To fix ideas, we consider the model problem

$$
\begin{array}{rlrl}
-\Delta u & =f, & & \text { in } \Omega \subset \mathbb{R}^{2}, \\
u=0, & & \text { on } \partial \Omega .
\end{array}
$$

Any discretization of (1) leads to a linear system of equations of the form

$$
A \mathbf{y}=\mathbf{f}
$$

where $\mathbf{y}$ is the vector of degrees of freedom representing approximations of $u$ and possibly $\nabla u$. A block Jacobi iteration with two non-overlapping subblocks is given by

$$
M \mathbf{y}^{(n+1)}=N \mathbf{y}^{(n)}+\mathbf{f}, \quad M=\left[\begin{array}{c|c}
A_{1} & O \\
\hline O & A_{2}
\end{array}\right], N=-\left[\begin{array}{c|c}
O & A_{12} \\
\hline A_{21} & O
\end{array}\right] .
$$

For classical discretizations of elliptic partial differential equations, like conforming finite elements or finite differences, block Jacobi methods are equivalent to classical Schwarz methods with minimal overlap, see for example [4]. This is different when the linear system (1) is obtained using DG methods.

Our paper is organized as follows: in section 2 we describe several DG methods for linear elliptic problems. We follow our discussion by introducing some "hybridizable" DG methods. In section 3 we show that block Jacobi iterations for the DG methods are corresponding to non-overlapping Schwarz methods with particular transmission conditions involving the penalty parameter of the DG method used. We then show numerical experiments in section 4, and present our conclusions in section 5 .

\section{Discontinuous Galerkin methods}

We introduce the so-called flux formulations, which define a class of discontinuous Galerkin methods for linear elliptic problems. We use the unified framework presented in [1].

Université de Genève, 2-4 rue du Lièvre, CP 64, CH-1211 Genève 4,

\{Martin.Gander, Soheil.Hajian\}@unige.ch 
Let $\mathscr{T}_{h}=\{K\}$ be a shape-regular triangulation of a polyhedral domain $\Omega \subset \mathbb{R}^{2}$. Let $h=\max _{K \in \mathscr{T}_{h}} h_{K}$. We denote by $\mathscr{E}^{0}$ the set of interior edges shared by all $K \in \mathscr{T}_{h}$, the set of boundary edges $\mathscr{E}^{\partial}$ and all edges by $\mathscr{E}:=\mathscr{E}^{\partial} \cup \mathscr{E}^{0}$.

Following [1] we define the broken Sobolev space $H^{l}\left(\mathscr{T}_{h}\right):=\prod_{K \in \mathscr{T}_{h}} H^{l}(K)$ and the trace space $T(\mathscr{E})=\prod_{K \in \mathscr{T}_{h}} L^{2}(\partial K)$ where $H^{l}(K)$ is the Sobolev space in $K \in \mathscr{T}_{h}$. We also define two trace operators: let $q \in T(\mathscr{E})$ and $\varphi \in[T(\mathscr{E})]^{2}$. On $e=\partial K_{1} \cap \partial K_{2}$ we then define average $\{\{\cdot\}\}$ and jump $[\cdot]]$ operators by

$$
\begin{aligned}
\{\{q\}\}=\frac{1}{2}\left(q_{1}+q_{2}\right), & \llbracket q \rrbracket]=q_{1} \mathbf{n}_{1}+q_{2} \mathbf{n}_{2}, \\
\{\{\varphi\}\}=\frac{1}{2}\left(\varphi_{1}+\varphi_{2}\right), & \llbracket \varphi \rrbracket]=\varphi_{1} \cdot \mathbf{n}_{1}+\varphi_{2} \cdot \mathbf{n}_{2},
\end{aligned}
$$

where $\mathbf{n}_{i}$ is the outward normal of $K_{i}$ on $e, q_{i}:=\left.q\right|_{\partial K_{i} \cap e}$ and $\varphi_{i}:=\left.\varphi\right|_{\partial K_{i} \cap e}$. On the boundary of $\Omega$ we set the average and jump operators to be $\{\{\varphi\}\}=\varphi$ and $[q q]=q \mathbf{n}$ respectively. We do not need to define $\{\{q\}\}$ and $\llbracket \varphi \rrbracket$ on $e \in \mathscr{E} \partial$; see [1].

We denote two finite dimensional broken spaces on $\mathscr{T}_{h}$ for the discrete approximation by $V_{h}:=\left\{v \in L^{2}(\Omega)\right.$ s.t. $\left.\left.v\right|_{K} \in P(K), \forall K \in \mathscr{T}_{h}\right\}$ where $P(K)=\mathbb{P}_{k}(K)$ and $\Sigma_{h}:=\left\{\tau \in\left[L^{2}(\Omega)\right]^{2}\right.$ s.t. $\left.\left.\tau\right|_{K} \in \Sigma(K), \forall K \in \mathscr{T}_{h}\right\}$ where $\Sigma(K)=\left[\mathbb{P}_{k}(K)\right]^{2}$. Here $\mathbb{P}_{k}(K)$ is the space of polynomials of degree $\leq k$ in the simplex $K \in \mathscr{T}_{h}$.

For the sake of simplicity we denote the volume and surface integrals by $(a, b)_{K}=$ $\int_{K} a b$ for $K \in \mathscr{T}_{h}$ and $\langle a, b\rangle_{e}=\int_{e} a b$ for $e \in \mathscr{E}$. Moreover $\|v\|_{0, \mathscr{T}_{h}}^{2}:=\sum_{K \in \mathscr{T}_{h}}(v, v)_{K}$.

\subsection{Flux formulation}

For the Laplacian model problem (1) in the DG context, one first rewrites the equation in mixed form,

$$
\sigma=\nabla u, \quad-\nabla \cdot \sigma=f(x), \quad x \in \Omega .
$$

Then the flux formulation is the following: let $K \in \mathscr{T}_{h}, v \in P(K)$ and $\tau \in \Sigma(K)$. We multiply (5) by $\tau$ and $v$ respectively. Integrating by parts over $K$, we substitute boundary terms of $u$ and $\sigma$ by two approximation functions. Hence the discrete weak form reads: find $\left(u_{h}, \sigma_{h}\right) \in V_{h} \times \Sigma_{h}$ for all $K \in \mathscr{T}_{h}$ such that

$$
\begin{aligned}
\left(\sigma_{h}, \tau\right)_{K} & =-\left(u_{h}, \nabla \cdot \tau\right)_{K}+\left\langle\hat{u}_{h}, \tau \cdot \mathbf{n}_{K}\right\rangle_{\partial K} & & \forall \tau \in \Sigma(K), \\
\left(\sigma_{h}, \nabla v\right)_{K} & =(f, v)_{K}+\left\langle v, \hat{\sigma}_{h} \cdot \mathbf{n}_{K}\right\rangle_{\partial K} & & \forall v \in P(K),
\end{aligned}
$$

where $\mathbf{n}_{K}$ is the outward normal of element $K$ and

$$
\hat{u}_{h}: H^{2}\left(\mathscr{T}_{h}\right) \times\left[H^{1}\left(\mathscr{T}_{h}\right)\right]^{2} \rightarrow T(\mathscr{E}), \quad \hat{\sigma}_{h}: H^{2}\left(\mathscr{T}_{h}\right) \times\left[H^{1}\left(\mathscr{T}_{h}\right)\right]^{2} \rightarrow[T(\mathscr{E})]^{2},
$$

which are called numerical fluxes. They approximate the traces of $u_{h}$ and $\sigma_{h}$ on $\partial K$. By defining $\hat{u}_{h}$ and $\hat{\sigma}_{h}$ we complete the definition of a DG method.

For instance we introduce the local discontinuous Galerkin method (LDG) with 


$$
\begin{aligned}
& \hat{u}_{h}=\left\{\left\{u_{h}\right\}\right\}-\beta \cdot\left[\left[u_{h}\right]\right] \text { on } \mathscr{E} 0, \quad \hat{u}_{h}=0 \text { on } \partial \Omega, \\
& \hat{\sigma}_{h}=\left\{\left\{\sigma_{h}\right\}\right\}+\beta\left[\left[\sigma_{h}\right]-\mu\left[\left[u_{h}\right]\right] \text { on } \mathscr{E},\right.
\end{aligned}
$$

where $\beta \in\left[L^{2}(\mathscr{E})\right]^{2}$ is a constant vector-valued function with $\beta=0$ on $\partial \Omega$ and $\mu \propto h_{e}^{-1}$ where $h_{e}$ is the edge length. We will consider the case $\beta=-\mathbf{n}_{K_{1}} / 2$ on $e=\partial K_{1} \cap \partial K_{2}$ where $K_{1}, K_{2} \in \mathscr{T}_{h}$ and the assignment of $\mathbf{n}_{K_{1}}$ is arbitrary. Therefore the numerical fluxes are

$$
\hat{u}_{h}=\left(u_{h}\right)_{K_{1}}, \quad \hat{\sigma}_{h}=\left(\sigma_{h}\right)_{K_{2}}-\mu \llbracket u_{h} \rrbracket \text { on } e .
$$

In case we have non-homogeneous Dirichlet data, e.g. $u=g_{D}$ on $\partial \Omega$, the numerical fluxes are

$$
\hat{u}_{h}=g_{D}, \quad \hat{\sigma}_{h}=\sigma_{h}-\mu\left(u_{h}-g_{D}\right) \text { on } e \in \mathscr{E}^{\partial} .
$$

We now introduce two more methods which are "hybridizable". A hybrid method is defined by eliminating interior unknowns within an element $K \in \mathscr{T}_{h}$ in terms of some unknowns defined on $\mathscr{E}^{0}$, called $\lambda_{h}$ (which here is $\hat{u}_{h}$ ). We then obtain a system for $\lambda_{h}$ which is much smaller than the original system. We do not derive these type of DG methods here but for a unified approach we refer the reader to [2].

Remark 1. A "hybridizable" DG method is designed to approximate the following continuous problem using $\hat{u}_{h}$ as Dirichlet data on $\partial K$ :

$$
\sigma-\nabla u=0 \text { and }-\nabla \cdot \sigma=f \text { in } K, \quad u=\hat{u}_{h}(u, \sigma) \text { on } \partial K .
$$

More precisely, their numerical fluxes are such that $\hat{\sigma}_{h}=\left(\sigma_{h}\right)_{K}-\mu\left[\left(u_{h}\right)_{K}-\hat{u}_{h}\right]$ on $\partial K$ which is the numerical flux one uses to impose Dirichlet boundary data on the boundary of an element, see (9).

We introduce two hybridizable methods, namely LDG-H and IP-H, by defining their numerical fluxes. The LDG-H uses

$$
\begin{aligned}
& \hat{u}_{h}=\frac{\mu_{1}}{\mu_{1}+\mu_{2}} u_{h, 1}+\frac{\mu_{2}}{\mu_{1}+\mu_{2}} u_{h, 2}-\frac{1}{\mu_{1}+\mu_{2}}\left[\sigma_{h}\right], \\
& \left.\hat{\sigma}_{h}=\frac{\mu_{2}}{\mu_{1}+\mu_{2}} \sigma_{h, 1}+\frac{\mu_{1}}{\mu_{1}+\mu_{2}} \sigma_{h, 2}-\frac{\mu_{1} \mu_{2}}{\mu_{1}+\mu_{2}}\left[u_{h}\right]\right],
\end{aligned}
$$

where $\mu \in T(\mathscr{E})$. Similarly for IP-H we have

$$
\begin{aligned}
& \hat{u}_{h}=\frac{\mu_{1}}{\mu_{1}+\mu_{2}} u_{h, 1}+\frac{\mu_{2}}{\mu_{1}+\mu_{2}} u_{h, 2}-\frac{1}{\mu_{1}+\mu_{2}}\left[\nabla u_{h}\right], \\
& \left.\hat{\sigma}_{h}=\frac{\mu_{2}}{\mu_{1}+\mu_{2}} \nabla u_{h, 1}+\frac{\mu_{1}}{\mu_{1}+\mu_{2}} \nabla u_{h, 2}-\frac{\mu_{1} \mu_{2}}{\mu_{1}+\mu_{2}} \llbracket\left[u_{h}\right]\right] .
\end{aligned}
$$

One can show that IP-H and LDG-H satisfy Remark 1 by noting that for $K \in \mathscr{T}_{h}$

$$
\hat{\sigma}_{h}=\left(\sigma_{h}\right)_{K}-\mu\left[\left(u_{h}\right)_{K}-\hat{u}_{h}\right] \text { on } \partial K .
$$




\section{Domain decomposition for "hybridizable" DG methods}

We decompose the domain $\Omega$ into two non-overlapping subdomains, $\left\{\Omega_{1}, \Omega_{2}\right\}$, such that the interface $\Gamma^{I}:=\bar{\Omega}_{1} \cap \bar{\Omega}_{2}$ is a subset of $\mathscr{E} 0$, i.e. the cut does not go through any element of $\mathscr{T}_{h}$. Therefore we obtain $\mathscr{T}_{h, 1}, \mathscr{T}_{h, 2}$ from the original $\mathscr{T}_{h}$, and similarly $\mathscr{E}_{1}^{0}, \mathscr{E}_{2}^{0}$, for our subdomains; see for example Fig. 1 (right).

Let $\left(u_{h}, \sigma_{h}\right)$ be the approximate solution obtained from a DG method. Let $\left(u_{h, 1}, \sigma_{h, 1}\right)$ be the restriction of $\left(u_{h}, \sigma_{h}\right)$ to $\Omega_{1}$ and similarly $\left(u_{h, 2}, \sigma_{h, 2}\right)$ to $\Omega_{2}$. Then $\left(u_{h, i}, \sigma_{h, i}\right)$ for $i=1,2$ and $K \in \mathscr{T}_{h, i}$ satisfy

$$
\begin{cases}\left(\sigma_{h, i}, \tau\right)_{K}=-\left(u_{h, i}, \nabla \cdot \tau\right)_{K}+\left\langle\hat{u}_{h, i}, \tau \cdot \mathbf{n}_{K}\right\rangle_{\partial K} & \forall \tau \in \Sigma(K), \\ \left(\sigma_{h, i}, \nabla v\right)_{K}=(f, v)_{K}+\left\langle v, \hat{\sigma}_{h, i} \cdot \mathbf{n}_{K}\right\rangle_{\partial K} & \forall v \in P(K),\end{cases}
$$

where

$$
\hat{u}_{h, i}:= \begin{cases}\hat{u}_{h}\left(u_{h, i}, \sigma_{h, i}, u_{h, j}, \sigma_{h, j}\right) & \text { on } \Gamma^{I} \text { and } j \neq i \\ \hat{u}_{h}\left(u_{h, i}, \sigma_{h, i}\right) & \text { on } \mathscr{E}_{i}^{0}\end{cases}
$$

and similarly for $\hat{\sigma}_{h, i}$. Note that we do not need to define $\hat{u}_{h, 1}$ on $\mathscr{E}_{2}^{0}$ since for $\left(u_{h, 1}, \sigma_{h, 1}\right)$ we only have one term in (14) that needs the trace of $\left(u_{h, 2}, \sigma_{h, 2}\right)$ on $\Gamma^{I}$ and not $\mathscr{E}_{2}^{0}$ (similarly $\hat{u}_{h, 2}$ does not need to be defined on $\mathscr{E}_{1}^{0}$ ).

If the trace of $\left(u_{h, 2}, \sigma_{h, 2}\right)$ is known on $\Gamma^{I}$, one can solve for $\left(u_{h, 1}, \sigma_{h, 1}\right)$ in $\Omega_{1}$, and vice versa. This suggests an iterative algorithm for solving $\left(u_{h, i}, \sigma_{h, i}\right)$ in parallel, namely: find $\left(u_{h, i}^{(n+1)}, \sigma_{h, i}^{(n+1)}\right)$ for $i=1,2$ such that it satisfies (14) with

$$
\hat{u}_{h, i}:= \begin{cases}\hat{u}_{h}\left(u_{h, i}^{(n+1)}, \sigma_{h, i}^{(n+1)}, u_{h, j}^{(n)}, \sigma_{h, j}^{(n)}\right) & \text { on } \Gamma^{I} \text { and } j \neq i \\ \hat{u}_{h}\left(u_{h, i}^{(n+1)}, \sigma_{h, i}^{(n+1)}\right) & \text { on } \mathscr{E}_{i}^{0}\end{cases}
$$

starting with an initial guess $\left(u_{h, i}^{(0)}, \sigma_{h, i}^{(0)}\right), i=1,2$. Note that $\hat{u}_{h, 1}$ is is not equal any more to $\hat{u}_{h, 2}$ on $\Gamma^{I}$ except at convergence, and then we have $\left(u_{h, i}^{\star}, \sigma_{h, i}^{\star}\right)=\left(u_{h, i}, \sigma_{h, i}\right)$, i.e. the domain decomposition approximation at convergence is equal to the mono domain approximate solution.

Denoting the degrees of freedom associated with $\left(u_{h, i}^{(n+1)}, \sigma_{h, i}^{(n+1)}\right)$ by $\mathbf{y}_{i}^{(n+1)}=$ $\left(\underline{u}_{i}{ }^{(n+1)}, \underline{\sigma}_{i}{ }^{(n+1)}\right)^{T}$ after choosing a basis for $P(K)$ and $\Sigma(K)$, we can write the equivalent linear systems for our iterative method as

$$
A_{1} \mathbf{y}_{1}^{(n+1)}=-A_{12} \mathbf{y}_{2}^{(n)}+\mathbf{f}_{1}, \quad A_{2} \mathbf{y}_{2}^{(n+1)}=-A_{21} \mathbf{y}_{1}^{(n)}+\mathbf{f}_{2},
$$

where $A_{12}$ is obtained from $\left\langle\hat{u}_{h, 1}, \tau \cdot \mathbf{n}_{K}\right\rangle_{e},\left\langle\hat{\sigma}_{h, 1} \cdot \mathbf{n}_{K}, v\right\rangle_{e}$ for $e \subset \Gamma^{I}$ and $A_{1}$ is the stiffness matrix obtained from (14) in $\Omega_{1}$, and similarly for $\Omega_{2}$. Setting $\mathbf{y}^{(n+1)}:=$ $\left(\mathbf{y}_{1}^{(n+1)}, \mathbf{y}_{2}^{(n+1)}\right)^{T}$ and $\mathbf{f}:=\left(\mathbf{f}_{1}, \mathbf{f}_{2}\right)^{T}$, we obtain precisely a block Jacobi iteration of the form (3).

For the classical finite element method with $\mathbb{P}_{1}$ approximation a block Jacobi iteration corresponds to a Schwarz method with minimal overlap and Dirichlet trans- 
mission conditions [4]. We show now that for hybridizable DG methods the block Jacobi iteration corresponds to a general Schwarz method of the form

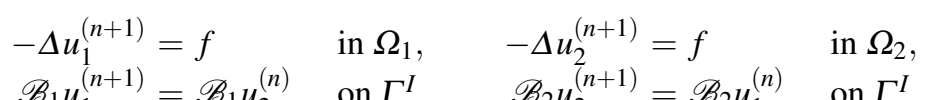

where $\mathscr{B}_{1}$ and $\mathscr{B}_{2}$ are two linear operators determined by the particular choice of DG discretization. The following propositions show the transmission condition on $\Gamma^{I}$ in (18), when $M$ and $N$ in (3) are obtained from LDG-H, IP-H and minimal dissipation LDG methods.

Proposition 1. Let $K_{1} \in \mathscr{T}_{h, 1}, K_{2} \in \mathscr{T}_{h, 2}$ and $e=\bar{K}_{1} \cap \bar{K}_{2} \subset \Gamma^{I}$. If $M$ and $N$ in (3) are obtained from an LDG-H discretization, then the block Jacobi iteration (3) is the discrete version of (18) with $\mathscr{B}_{1}=\partial_{\mathbf{n}_{1}}+\mu_{2}$ and $\mathscr{B}_{2}=\partial_{\mathbf{n}_{2}}+\mu_{1}$ on e.

Proof. We start with $K_{1}$ : since the numerical fluxes of the LDG-H satisfy the condition in Remark 1, i.e. $\hat{\sigma}_{h, 1}=\sigma_{h, 1}^{(n+1)}-\mu_{1}\left(u_{h, 1}^{(n+1)}-\hat{u}_{h, 1}\right) \mathbf{n}_{1}$, one can conclude that we are imposing the following Dirichlet data at the continuous level: $u_{1}^{(n+1)}=\hat{u}_{h, 1}\left(u_{1}^{(n+1)}, \sigma_{1}^{(n+1)}, u_{2}^{(n)}, \sigma_{2}^{(n)}\right)$ on $e$. From the definition of the LDG-H numerical flux (11) we obtain

$$
u_{1}^{(n+1)}=\frac{\mu_{1}}{\mu_{1}+\mu_{2}} u_{1}^{(n+1)}+\frac{\mu_{2}}{\mu_{1}+\mu_{2}} u_{2}^{(n)}-\frac{1}{\mu_{1}+\mu_{2}}\left(\sigma_{1}^{(n+1)}-\sigma_{2}^{(n)}\right) \cdot \mathbf{n}_{1} .
$$

Collecting terms with super index $(n+1)$ and noting $\sigma_{i} \cdot \mathbf{n}_{1}=\partial_{\mathbf{n}_{1}} u_{i}$ on $e$, we obtain $\mathscr{B}_{1}=\partial_{\mathbf{n}_{1}}+\mu_{2}$. The same argument applies to $K_{2}$.

Proposition 2. Let $K_{1} \in \mathscr{T}_{h, 1}, K_{2} \in \mathscr{T}_{h, 2}$ and $e=\bar{K}_{1} \cap \bar{K}_{2} \subset \Gamma^{I}$. If $M$ and $N$ in (3) is obtained from an IP-H discretization, then the block Jacobi iteration (3) is the discrete version of (18) with $\mathscr{B}_{1}=\partial_{\mathbf{n}_{1}}+\mu_{2}$ and $\mathscr{B}_{2}=\partial_{\mathbf{n}_{2}}+\mu_{1}$ on e.

Proof. This result can be proved similarly to the proof of Proposition 1.

Proposition 3. Let $K_{1} \in \mathscr{T}_{h, 1}, K_{2} \in \mathscr{T}_{h, 2}$ and $e=\bar{K}_{1} \cap \bar{K}_{2} \subset \Gamma^{I}$. Let $M$ and $N$ in (3) be obtained from a minimal dissipation $L D G$ and assume $\beta:=-\mathbf{n}_{1} / 2$, then the block Jacobi iteration (3) is the discrete version of (18) with $\mathscr{B}_{1}=\partial_{\mathbf{n}_{1}}+\mu_{2}$ and $\mathscr{B}_{2}=1$ on e.

Proof. We start with $K_{2}$ : note that with this definition of $\beta$ we have $\hat{u}_{h, 2}=u_{h, 1}^{(n)}$ and $\hat{\sigma}_{h, 2}=\sigma_{h, 2}^{(n+1)}-\mu_{2}\left(u_{h, 2}^{(n+1)}-u_{h, 1}^{(n)}\right) \mathbf{n}_{2}$. Comparing with (9), one concludes that we are imposing $u_{1}^{(n+1)}=u_{2}^{(n)}$ on $e$. Now for $K_{1}$ using the definition of $\hat{u}_{h, 1}=u_{h, 1}^{(n+1)}$ on $e$ in the first equation of (14) one obtains:

$$
\left(\sigma_{h, 1}^{(n+1)}-\nabla u_{h, 1}^{(n+1)}, \tau\right)_{K_{1}}=\left\langle\hat{u}_{h, 1}-u_{h, 1}^{(n+1)}, \tau \cdot \mathbf{n}_{1}\right\rangle_{\partial K_{1} \backslash e} \quad \forall \tau \in \Sigma\left(K_{1}\right) .
$$

Choosing $\tau=\nabla v$ (since $\nabla V\left(K_{1}\right) \subset \Sigma\left(K_{1}\right)$ ), substituting into the second equation of (14) yields 


$$
\begin{aligned}
\left(\nabla u_{h, 1}^{(n+1)}, \nabla v\right)_{K_{1}}= & \left\langle\hat{\sigma}_{h, 1} \cdot \mathbf{n}_{1}, v\right\rangle_{e}+(f, v)_{K_{1}} \\
& +\left[\left\langle\hat{u}_{h, 1}-u_{h, 1}^{(n+1)}, \tau \cdot \mathbf{n}_{1}\right\rangle_{\partial K_{1} \backslash e}+\left\langle\hat{\sigma}_{h, 1} \cdot \mathbf{n}_{1}, v\right\rangle_{\partial K_{1} \backslash e}\right] .
\end{aligned}
$$

Therefore one can conclude that the following Neumann boundary data is imposed on the interface: $\sigma_{1}^{(n+1)} \cdot \mathbf{n}_{1}=\hat{\sigma}_{h, 1}\left(u_{1}^{(n+1)}, \sigma_{1}^{(n+1)}, u_{2}^{(n)}, \sigma_{2}^{(n)}\right) \cdot \mathbf{n}_{1}$ on $e$. Using the definition of $\hat{\sigma}_{h, 1}()=.\sigma_{2}^{(n)}-\mu_{2}\left(u_{1}^{(n+1)}-u_{2}^{(n)}\right) \mathbf{n}_{1}$ and collecting terms with super index $(n+1)$ leads to $\mathscr{B}_{1}=\partial_{\mathbf{n}_{1}}+\mu_{2}$.

The results here are also applicable when a positive reaction terms is present, e.g. for $(\eta-\Delta) u=f, \eta>0$, since the zeroth order term only adds a term like $\eta(u, v)_{K}$ in the mixed formulation, and thus does not change numerical fluxes.

\subsection{Comments on optimized Schwarz methods for DG discretizations}

One can estimate the convergence of the block Jacobi method by analyzing the convergence behavior of the equivalent algorithm at the continuous level given in (18). This has been done for a simple geometry in [5], where for the case $\mu_{1}=\mu_{2}=: \mu$ on $\Gamma^{I}$, it is shown that the "uniformly optimal" value for $\mu$ is $\mu^{*}=\left(\left(K_{\min }^{2}+\eta\right)\left(K_{\max }^{2}+\eta\right)\right)^{\frac{1}{4}}$. Here $K_{\min }$ and $K_{\max }$ are the minimum and maximum frequencies that can be represented on the interface, heuristically chosen to be $K_{\min }=\pi$ and $K_{\max }=\frac{\pi}{h}$ for an interface of length one. Therefore $\mu^{*} \propto h^{-\frac{1}{2}}$. The contraction factor of the Fourier modes in (18) is then bounded by $\rho^{*}=1-O(\sqrt{h})$.

We have seen that for the DG methods presented the penalty parameter enters as Robin parameter in the equivalent continuous Schwarz method. The penalty parameter in DG methods is chosen such that it ensures coercivity of the bilinear form as well as optimal convergence of the discrete approximation to the continuous solution.

Here we would like to comment only for $L D G-H$ on how to choose $\mu$ such that one obtains optimal convergence to the continuous solution and achieves fast convergence of the block Jacobi iteration at the same time. For LDG-H, $\mu$ can be chosen as $O(1)$ or $O\left(h^{-1}\right)$. However using [3, Theorem 2.2], it can be shown that using $\mu \propto h^{-\frac{1}{2}}$ for a class of DG methods in which LDG-H is also included yields an optimal convergence to the continuous solution and we have the following corollary.

Corollary 1. Let the discretization be LDG-H and consider the domain decomposition setting in section 3. Set $\mu=h^{-\frac{1}{2}}$ on $\Gamma^{I}$ and $\mu=h^{-\alpha}$ for $0 \leq \alpha \leq 1$ on $\mathscr{E} \backslash \Gamma^{I}$. Then $\left\|u_{h}-u\right\|_{0, \mathscr{T}_{h}} \leq C h^{k+1}$, i.e. optimal approximation. Moreover the contraction factor of the iterative domain decomposition method (block Jacobi), is bounded by $\rho=1-O(\sqrt{h})$ which cannot be improved for any other choice of $\mu$ on $\Gamma^{I}$. 

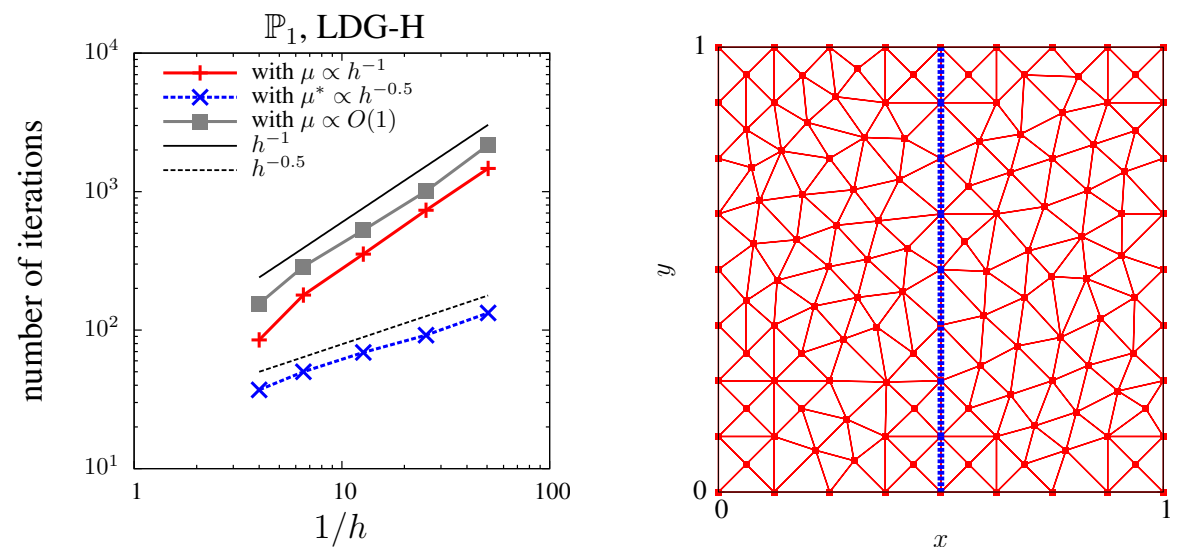

Fig. 1 (left) asymptotic number of iterations required by the non-overlapping Schwarz method using LDG-H. (right) unstructured mesh with the interface $\Gamma^{I}=\{0.5\} \times(0,1)$.

\section{Numerical experiments}

We consider $(\eta-\Delta) u=f$ in $\Omega$ and $u=0$ on $\partial \Omega$ where we set $\eta=1, \Omega=(0,1)^{2}$ and $f$ such that the exact solution is $u(x, y)=\sin (\pi x) \sin \left(2 \pi x+\frac{\pi}{4}\right) \sin (2 \pi y)$ in $\Omega$. We illustrate the results in section 3 using a block Jacobi method as in (3) with $\Gamma^{I}=\{0.5\} \times(0,1)$ as interface on an unstructured mesh; see Fig. 1 (right).

The penalty parameter is usually chosen as $\mu=k^{2} / h_{e}$ where $k$ is the degree of the polynomials; this would correspond to a very unusual high frequency approximation of the DtN operator in the optimized Schwarz method, and thus strongly affects the convergence rate. The convergence results in Fig. 2 are obtained by mea-
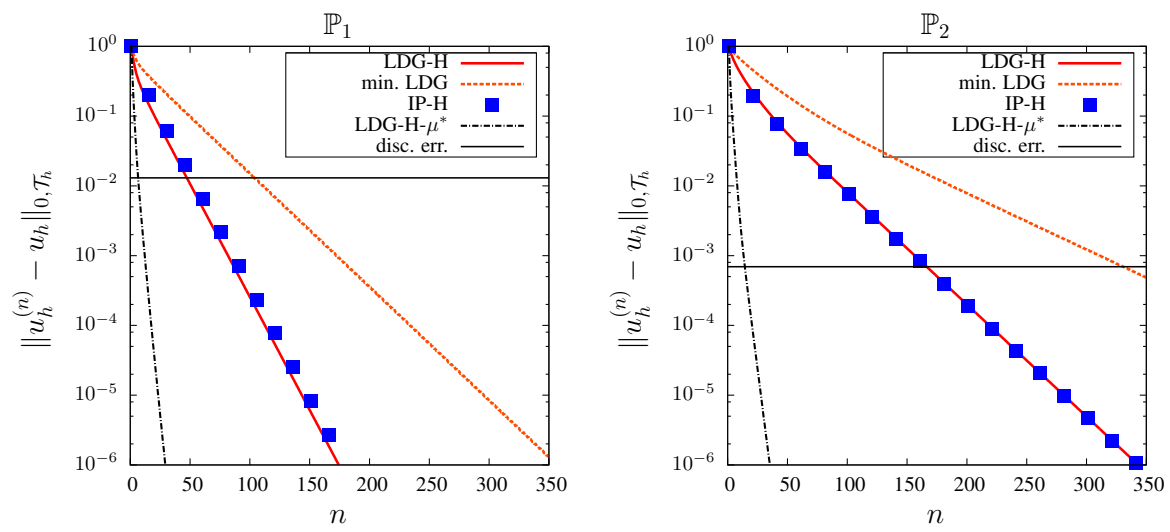

Fig. 2 Block Jacobi method for LDG-H, minimal dissipation LDG, IP-H, LDG-H with $\mu^{*}$ and discretization error for $\mathbb{P}_{1}$ and $\mathbb{P}_{2}$. 
suring $\left\|u_{h}^{(n)}-u_{h}\right\|_{0, \mathscr{T}_{h}}$, where $u_{h}$ is the mono-domain approximate solution and $u_{h}^{(n)}$ is the solution obtained at iteration $n$ of the block Jacobi method for $\mathbb{P}_{1}$ and $\mathbb{P}_{2}$. It is evident that IP-H and LDG-H converge faster than minimal dissipation LDG in the block Jacobi iteration due to their transmission conditions. Moreover LDG-H with $\mu^{*}$ converges faster than LDG-H using $\mu \propto h^{-1}$ since its parameter is chosen as suggested by optimized Schwarz framework.

Fig. 1 (left) shows the number of iterations required for the block Jacobi method to reduce the error to the machine precision for LDG-H with different penalty parameters on $\Gamma^{I}$ on a sequence of unstructured meshes. We show that for LDG-H the contraction factor with "uniformly" optimal $\mu^{*}$ behaves as predicted in Corollary 1 and [5], i.e. $\rho^{*}=1-O(\sqrt{h})$, while with $\mu=O(1)$ or $O\left(h^{-1}\right)$ behaves like $\rho=1-O(h)$.

\section{Conclusions}

We have shown that block Jacobi methods for DG discretizations correspond to non-overlapping optimized Schwarz methods with Robin-, or Robin and Dirichlet transmission conditions. This is in contrast to standard finite element methods, where block Jacobi methods correspond to classical Schwarz methods with minimal overlap and Dirichlet transmission conditions. In addition, we found that the penalty parameter in certain DG method leads to a high frequency approximation in the transmission condition of the optimized Schwarz method, which is not a very good choice for the convergence of the Schwarz method. We are currently studying a way to introduce a much better parameter for the convergence of block Jacobi, without changing however the DG approximation properties.

Acknowledgements We would like to thank BLANCA AYUSO for her useful comments.

\section{References}

1. Douglas N. Arnold, Franco Brezzi, et al. Unified analysis of discontinuous Galerkin methods for elliptic problems. SIAM J. Num. Anal., 39(5):1749-1779, 2002.

2. Bernardo Cockburn, Jayadeep Gopalakrishnan, and Raytcho Lazarov. Unified hybridization of discontinuous Galerkin, mixed, and continuous Galerkin methods for second order elliptic problems. SIAM J. Num. Anal., 47(2):1319-1365, 2009.

3. Paul Castillo, Bernardo Cockburn, et al. An a Priori Error Analysis of the Local Discontinuous Galerkin Method for Elliptic Problems. SIAM J. Num. Anal., 38(5):1676-1706, 2001.

4. M. J. Gander. Schwarz methods over the course of time. Electronic Transactions on Numerical Analysis, 31:228-255, 2008.

5. M. J. Gander. Optimized Schwarz Methods. SIAM J. Num. Anal., 44(2):699-731, 2006. 